\title{
Erratum to "Prediction of Treatment Response to Donepezil using Automated Hippocampal Subfields Volumes Segmentation in Patients with Mild Alzheimer's Disease"
}

\author{
Yoo Hyun Um¹, Tae-Won Kim¹, Jong-Hyun Jeong', Ho-Jun Seo', \\ Jin-Hee Han ${ }^{1}$, Seung-Chul Hong', Chang-Uk Lee ${ }^{2}$, and Hyun Kook Lim ${ }^{2} \bowtie$ \\ 'Department of Psychiatry, St. Vincent's Hospital, College of Medicine, The Catholic University of Korea, Suwon, Republic of Korea \\ ${ }^{2}$ Department of Psychiatry, Seoul St. Mary's Hospital, College of Medicine, The Catholic University of Korea, Seoul, Republic of Korea
}

Psychiatry Investig 2017;14(5):698-702

https://doi.org/10.4306/pi.2017.14.6.698

Unfortunately, the corresponding author's affiliation was incorrect in the original publication of this article. The correct affiliation is given below: Hyun Kook Lim, Department of Psychiatry, Yeouido St. Mary's Hospital, College of Medicine, The Catholic University of Korea, Seoul, Republic of Korea

Yoo Hyun Um¹, Tae-Won Kim¹, Jong-Hyun Jeong', Ho-Jun Seo', Jin-Hee Han', Seung-Chul Hong', Chang-Uk Lee ${ }^{2}$, and Hyun Kook Lim ${ }^{3 凶}$

1'Department of Psychiatry, St. Vincent's Hospital, College of Medicine, The Catholic University of Korea, Suwon, Republic of Korea ${ }^{2}$ Department of Psychiatry, Seoul St. Mary's Hospital, College of Medicine, The Catholic University of Korea, Seoul, Republic of Korea ${ }^{3}$ Department of Psychiatry, Yeouido St. Mary's Hospital, College of Medicine, The Catholic University of Korea, Seoul, Republic of Korea 